\title{
Why Groupware Succeeds: Discretion or Mandate?
}

\author{
Jonathan Grudin and Leysia Palen \\ University of California, Irvine, USA
}

\begin{abstract}
Single-user applications are designed with a 'discretionary use' model. In contrast, for large systems, upper management support is considered crucial to adoption. Which applies to groupware? The relatively low cost of groupware reduces high-level visibility, but some argue that social dynamics will force mandated use-the large system approach. Interview studies of recently adopted on-line meeting schedulers in two large organizations found successful, near-universal use achieved without managerial mandate. Versatile functionality and ease of use associated with discretionary products appeared to be factors leading to adoption. Other factors included organization-wide infrastructure and substantial peer pressure that developed over time.
\end{abstract}

\section{Introduction}

Many groupware developers hope to emulate the success of individual productivity software products such as word processors and spreadsheets. The use of singleuser applications is often discretionary, and they are designed to appeal to discretionary users. Even corporate purchasers who do not themselves use such a product may consult users. Discretionary use and competition have led to emphasis on ease of use and more functionality. In contrast, a large system built or tailored for a specific setting has fewer resources for minor features and interface tuning, and requires users to adjust more. A large system has a range of users, not all of whom may benefit from or like it. Studies of the introduction of large systems invariably report that strong backing by upper management is critical to success.

Groupware lies between the two. Similarities to large systems include the need to tailor to individual settings and the likelihood of mixed reactions by group 
members. It has been argued that groupware success will require mandated use. On the other hand, the low (and downward-spiraling) cost of groupware gives it less visibility in a large organization; it is unlikely that upper management will become involved in promoting every groupware application or feature.

For example, Markus and Connolly (1990) examined social factors in the adoption of groupware tools and concluded that mandated use or other top-down measures appeared necessary for the use of these tools to reach 'critical mass.' In a widely-cited case of groupware adoption (Orlikowski, 1992), a high-level mandate to use LOTUS NOTES received a mixed response, but use continued at Alpha Corp.

However, conflicting signs are present. An interesting aspect of Orlikowski's study is that the technical support staff, under no mandate to use NOTES, adopted it successfully. Markus (1995) studied mandated use of electronic mail in one organization and found that how email was used was highly discretionary and varied. And of course the use of email often spreads without a high-level mandate, although email is a risky application from which to generalize about groupware.

By examining factors that underlie cases of successful groupware adoption, our study addresses the tension between the recognized importance of high-level support for large system adoption and the lower visibility groupware can expect.

We examined collaborative meeting schedulers, applications with recent successes that stand in contrast to early, documented failures. About 15 years ago, commercial office systems began to provide meeting scheduling features with electronic calendar applications. Computer-assisted meeting scheduling represented an appealing technical solution to a clearly identified problem: People spend a lot of time arranging and rearranging meetings. Nevertheless, it was difficult to find successful use of the software, even in the development organizations and research laboratories that produced the software and provided widespread access to it. In fact, Grudin (1988) used meeting scheduling to illustrate several factors that contribute to the lack of widespread adoption of some groupware applications.

Today, successful use is more widespread. Electronic meeting scheduling occurs in environments very similar to those where it was not found a decade ago. In this paper we review the findings of the mid-1980s, then describe two detailed interview studies conducted in environments similar in many respects to those examined a decade earlier, but where on-line meeting scheduling is now routine.

The settings we studied are not typical, and we do not claim that the software will soon be universally adopted. Quite the contrary, the settings are special: they are software development organizations. However, meeting scheduling was not adopted in comparable settings a decade earlier. What has changed in the software or in these environments that contributes to current use? What is the adoption process? The actual or potential presence in other settings of such factors, once identified, might signal the usefulness of this or other groupware and suggest adoption processes to expect or encourage. 


\section{Meeting scheduling in 1985}

By the early 1980s, many organizations had installed office systems that provided word processing, spreadsheets, email, and on-line calendars. In settings in which on-line calendars are widely available, the concept of computer-assisted meeting scheduling is simple: A person scheduling a meeting identifies the participants and the application checks each person's calendar, finds a time when everyone is free, and schedules a meeting or issues invitations. The potential utility was clear: The time spent scheduling and rescheduling meetings is substantial. Opportunities lost through inefficiency in meeting scheduling had been identified (Ehrlich, 1987a).

However, a study conducted in a large organization that developed and marketed an early electronic calendar identified factors that contributed to a lack of use of the meeting scheduling feature (Ehrlich, 1987a; 1987b; Grudin, 1988). Electronic calendars were used as communication devices by executives, managers, and their secretaries, but only by about one in four individual contributors. The latter, if they kept any calendar at all, found portable paper calendars more congenial-available in meetings, for example. To maintain an on-line calendar would require more work of individual contributors, but the direct beneficiaries would be the managers and secretaries who called most meetings. In addition, although most employees had computer access, not everyone in the organization was networked tightly enough for the software to reach them. As a result, meetings were scheduled by traditional methods, despite the presence of the software on everyone's desks.

Research papers at early CSCW conferences described new approaches to meeting scheduling that did not fare much better. Woitass (1990) designed a system to address the access problem. Based on a distributed agent architecture, it consulted the electronic calendars of those maintaining them and engaged other users directly. Although a technical success, Woitass reported that the system was not used for much the same reason: It required work from those who did not see the benefit of using it. Beard et al. (1990) developed a priority-based visual calendaring system that performed well in controlled experiments, but in field tests encountered a range of problems, most notably a lack of integration with users' desktop applications. In their presentation, they suggested that these applications would become fully useful when portable computers eliminate some advantages of paper calendars. Finally, a 1991 survey of groupware use was directed at Internet distribution lists of "empirical researchers, system developers, and end-users of CSCW products and systems" (Butterfield, Rathnam, and Whinston, 1993). Respondents indicated that group calendaring systems were the most widely available of 10 groupware technologies by a substantial margin, but were judged to be the least likely to have a significant impact.

Collaborative meeting schedulers are not unique in requiring additional work (in this case calendar maintenance) from people who do not see a benefit in their use. Most large systems and major applications face this challenge when introduced. 
But an important difference is that groupware is often not a large, expensive system: It can be a small application or even a feature. The collective benefit of using a feature or small application is not as evident, it does not have the visibility in the organization that an expensive system does, so it is unlikely to get the same degree of management support. To promote a large system, management may hire support staff such as system administrators and data entry personnel, change staff job descriptions, and encourage or even mandate use. In contrast, managers in the environments examined in the 1980s did not mandate that individual contributors maintain on-line calendars solely to facilitate meeting scheduling, nor were secretaries asked to maintain the on-line calendars of individual contributors.

Upper management advocacy is a key element in large system adoption. In contrast, the use of individual productivity tools or single-user applications has more often been discretionary; an application must offer each user a tangible reward. Groupware is caught in the middle: It often must appeal to all group members, yet can expect little in the way of top-down advocacy.

But the situation was never deemed hopeless: "The conclusion is not entirely negative. Automatic meeting scheduling could be targeted to environments or groups making the most uniform use of electronic calendars. Their value can be enhanced by adding conference room and equipment scheduling..." (Grudin, 1988). "Focus on improving the interface for those who receive less direct benefit... this may seem obvious but is very difficult" (Grudin and Poltrock, 1990).

It is difficult to focus interface design on "incidental" users because an application must appeal to its principal beneficiaries. A meeting scheduler that does not appeal to the manager or secretary who calls meetings has no chance. Interface development is usually directed toward those who will benefit most from using an application, not those who benefit least (Grudin, 1994).

Ehrlich's (1987a) data suggested one way to target existing calendar users. She reported a higher incidence of secretary-supported calendar use among managers and executives in the development organization she studied. Electronic support for scheduling meetings of managers was therefore feasible, and is often reported.

"PROFS (Office Vision) is the single most used office system in the company. Most people with PROFS accounts do not use its calendar. Almost all managers/supervisors use the calendar, and secretaries maintain the calendar for 2 nd level managers and higher... They collected data in (a group of primarily managers and secretaries) and found very large time savings and cost savings due to the online calendar." (Steven Poltrock, personal communication, 10/3/94.)

Obstacles hinder the wider use of this particular system:

"In my [group] most people use an online calendar, but our calendars are not shared - we are not using PROFS.” (Ibid.)

Our study involves engineering environments in which scheduling is not restricted to managers, engineering environments similar to those in which meeting scheduled failed in 1985. The prescription to improve interfaces for individual contributors is part of what we found has changed over the years, although it is not the full story. 


\section{Meeting scheduling in 1995}

Although meeting scheduling is far from universal or even widespread-it not used in the authors' workplace, for example-reports of its use in engineering environments are encountered with growing frequency, even in the absence of portable computing. Questions prompted by these reports include:

- How widespread is calendaring and meeting scheduling at these sites?

- Who uses them?

- Are new application functionality and/or interfaces factors?

- Has the collective benefit of use been made more salient?

- Is adoption the result of top-down encouragement or mandate?

- How does adoption proceed over time?

\section{Site Descriptions}

\section{Microsoft Corporation}

Most of Microsoft's 15,000 employees are located in the Seattle area, where the interviews were conducted. The average employee age is 34 , low for a large software company. For many of them, Microsoft is their sole or principal source of work experience. Most employees in the development divisions have their own office or share with one other person, and every employee has ready access to a common platform computer that is linked to the company-wide network.

\section{Sun Microsystems}

About half of Sun Microsystems' 13,000 employees work in the (San Francisco) Bay Area, with about 3,000 in the engineering departments where our interviews took place. The average age of Sun employees is 38. Each has an office or shares with one person, except for admins who are typically in cubicles in central locations. Employees have workstations connected to the company-wide network. ("Admins," short for "administrators" or "administrative assistants," are responsible for secretarial and other coordination functions.)

\section{Method}

The second author spent three months as a participant observer in a Microsoft development division, at which time she conducted five interviews on the topic of meeting scheduling. Based on experiential data and the Microsoft interviews, we developed a set of 40 questions that she used in twelve interviews conducted over a 3-day period at Sun. We had learned of widespread use of scheduling at these sites and sought active users. Our interest was in identifying possible success factors; the specific adoption contrast that frames this paper emerged during the analysis. 
Participants

Microsoft. The five interviews were of people with different job titles across the development divisions. Each participant was in a different workgroup. All had been employed at Microsoft before the electronic calendar application was introduced, with an average of about 5 years' service. Listed in increasing order of time spent in meetings, the subjects were a developer, visual designer, program manager (project leader), development instructor and first level manager.

Sun. Eleven people from ten different workgroups in engineering organizations and one person in corporate marketing were interviewed. They had been at Sun over five years on average. Two arrived after the electronic calendar was introduced. Eleven now use the electronic calendar; the twelfth once used it but no longer does. Three participants were or had been on the CALENDAR MANAGER product team and provided technical implementation information, an historical account of the product's introduction, and data about their personal use. Listed in roughly increasing order of time spent in meetings: two administrative personnel, two technical writers, three developers, two human interface designers, one product marketer, one manager, and one corporate marketer.

Interview questions

We queried subjects about their use of paper and electronic calendars. Questions covered procedures and protocols for arranging or responding to meeting invitations, privacy, granting viewing access, peer pressure, managing one's calendar when away from the computer, using calendars for structuring work, and using calendars for handling resources such as conference rooms and equipment.

\section{Description of the Electronic Calendar Applications}

We next describe features of the two electronic calendars. These applications are maturing into versatile, flexible systems with a wide range of capabilities and multiple paths for achieving many goals. Readers familiar with them may prefer to skip to the discussion.

\section{Microsoft's SCHEDULE+ ${ }^{T M}$}

SCHEDULE+ runs on PCs under WINDOWS. Individual calendars are kept on the owners' machines with frequent updates to a central server. Users view a calendar by specifying its owner's email alias or name, the latter a recent enhancement.

Viewing the Calendar. SCHEDULE+ supports two on-screen calendar views. The day view provides complete textual information about each appointment and symbols that indicate if the appointment is a recurring meeting, a "locked" meeting not viewable by others, a tentative meeting, or a tentative meeting contingent on others agreeing. The planner view provides a quick check for availability over 17 days, with booked times blocked out graphically and no appointment content detail. 
For printing, SCHEDULE+ supports day, week, and month views as well as a textual list of appointments. Printed views are designed to be put in appointment books and thus differ in appearance from on-screen views.

Navigation. Drop down menus allow users to jump to a specific day or week. Clicking on a specific day in a small monthly calendar graphic in a corner of the day view jumps directly to that day. Arrows enable one to advance by month or year.

Privacy Settings. Users can allow their calendars to be viewed by anyone in the company, by certain people, or by no one. Additionally, users can determine what level of access another person has: restricted viewing reveals when time is booked or free, but not the content of appointments; complete viewing; and "assistant" privilege, which allows another person to accept and send meeting requests on behalf of the calendar owner. Calendar owners can "lock" a meeting, making it completely private.

Booking Appointments. One way to book appointments is to select the time and duration in the day view, then type the description of the event directly into the view. Another way is to bring up the "Edit Appointment" dialogue box (either by clicking on a time or using menus), which provides input fields for appointment information, including whether a meeting is to recur every day, every work day, once a week, once a month, or in one of several other patterns. The dialogue window also allows one to set the lead time on alerts: a pop-up window and/or an audible reminder of an imminent appointment.

Group appointments are booked in one of two ways. 1) The meeting arranger enters the appointment details in the Edit Appointment dialogue box, then invites other people by specifying either their email aliases or full names. (An on-line rolodex tool can help locate names and aliases.) This spawns another window with a mini-planner view where the invitees' calendars are overlaid to reveal available times. Clicking on a booked slot causes a symbol to appear next to the email aliases of those who are booked at that time. 2) These steps can be reversed: The meeting arranger first specifies the people to attend, determines an available time from the overlaid calendars, and then enters the appointment details. This flexibility in sequencing allows meeting scheduling to proceed naturally in circumstances that vary regarding the availability of attendees, the importance of a meeting, and whether key people must be present.

Both scheduling procedures typically end with the meeting arranger initiating email from within SCHEDULE+. All attendees' email addresses appear in the To field, the meeting topic in the Subject field, and the meeting time in a special When field. The meeting arranger can also check a Send Response box, which generates response mail based on attendees' decisions to accept or decline the meeting. The meeting arranger can then track the status of the responses within another SCHEDULE+ dialogue window.

Receiving SCHEDULE+ Mail. An email message generated within SCHEDULE+ arrives with regular email. The message header includes Accept, Decline, Tentative, and View Schedule buttons. Selecting View Schedule launches 
SCHEDULE+ (if it is not already open) to enable the recipient to review his or her schedule to confirm availability. If the recipient selects Accept, the meeting is automatically entered into his or her calendar and a mail window is generated to reply to the sender with "Yes, I will attend" as part of the header. If Decline is selected, a mail window is opened with "No, I will not attend" in the message header. Tentative produces the message "I might attend." With any of these, a responder can include additional information in the message body.

\section{SUn's CALENDAR MANAGER ${ }^{T M}$}

CALENDAR MANAGER runs under OPENWINDOWS and CDE on SPARCSTATIONS. When open but inactive, it appears as an icon showing the current date. Calendars are maintained on their owner's host machines. Browsing other calendars requires specifying the host on which each calendar resides. Host names are listed in an online corporate rolodex of employee information.

Viewing the Calendar. CALENDAR MANAGER supports identical on-line and printed day, week, month, and year views, the latter including no appointment information. The day view presents meeting information in hour slots and provides small depictions of the current and next two months for navigation and context. In the week view, the view preferred by most employees interviewed, a full week is displayed two ways. Each day appears as a pane containing a simple text list of the day's appointments, including start times, end times and appointment details. In addition, the week is displayed with booked and free times shown graphically for an at-a-glance assessment. Appointments are shaded; overlapping appointments appear as progressively darker shading. The month view displays a given month with reduced appointment information.

Navigation. In the day view, advance and reverse buttons shift one day at a time. Clicking in one of the small month depictions jumps ahead. Similar buttons in the week and month views advance or go back a week or month. Users can thus jump to a week in a distant month by switching from week to month views, clicking to reach the desired month, and then returning to the week view.

Privacy Settings. As with SCHEDULE+, multiple levels of view access can be set for different appointments at the calendar owner's discretion. CALENDAR MANAGER specifies the same three levels of viewing access: Show Time and Text, Show Time Only, and Show Nothing. A global default is set and then overwritten as desired for specific appointments.

Privileges can also be assigned to specific people. Browse allows an assigned person to view one's calendar, with the viewable content level dependent on the access level assigned to each appointment. Insert allows a person to insert meetings into the owner's calendar and delete the same meetings later if necessary. Delete privilege allows a person to delete any meetings in the owner's calendar.

Booking Appointments. Appointments are booked by initiating the Appointment Editor dialogue box, either with a pull-down menu, by clicking on the desired meeting time in the day view, or by selecting the desired day in the week, month, or 
year views. The Appointment Editor has fields for the date, start and end times and a general content field for the subject and location. Privacy default settings can be modified and auto-reminders can be set in this dialogue box. Auto-reminder options are a pop-up window, beeping, screen flash, and sending email (typically to oneself, although any email alias can be specified for reminding). Any combination of auto-reminders with independently set warning intervals can be used, although a global default eliminates the need to set options for each appointment. Finally, appointments can be set to recur at a variety of time intervals.

Checking for Availability. There are several ways to arrange meetings, combining automated features and social protocols. The Multibrowser can be invoked to determine the availability of attendees. It overlays calendars that are specified by email alias and host machine name. Again, shaded time indicates unavailability; the darker the shading, the more people are unavailable. Once the Multibrowser is invoked, there are several ways to proceed. The meeting arranger can select an available time, enter the meeting details and then send email from within CALENDAR MANAGER by selecting the Mail button on the Multibrowser. This generates a Mail Tool window with invitees in the To field and an appointment icon in the attachments section of the mail message. Recipients can drag this icon to their calendars to automatically insert meeting and content information.

Another way to proceed is to use the Multibrowser to find two or three possible times and use the Mail Tool (outside CALENDAR MANAGER) initiate normal email, asking invitees to choose among the possibilities. The arranger then chooses a time based on the responses and sends an official announcement, either as regular email, or from within the calendar to automatically include an appointment icon. Other ways to send the final notification through regular email are to drag appointment information from the Appointment editor and drop it into the email message or copy a template into the regular email and manually type in the fields.

Receiving Mail with CALENDAR MANAGER Information. Meeting invitees who receive email enclosing an appointment icon attachment or a template can drag it to their calendars for automatic entry. These attachments or templates are used for notification of large meetings ("all hands"), by administrators to distribute company holiday lists, and by meeting arrangers who confirm a meeting time by negotiating the time first and then sending automated information as a convenience to the invitees and to ensure that everyone has the same information.

\section{Overview of Use}

We had sought informants who used on-line meeting scheduling, so it was not surprising that high usage was reported in 16 of the 17 interviews (i.e., 15 of the 16 groups). The exception was the Sun corporate marketing representative, a former user, who frequently met with external contacts, people not accessible through the system. 
At Microsoft, the most commonly used privacy setting was the application default, restricted viewing. This allows others to see booked and free time but not the details of scheduled appointments. People typically schedule a meeting by checking the availability of others and sending SCHEDULE+ mail with a proposed time, allowing the recipients to accept or decline with a keystroke. This approach, with no informal prior negotiation of time, can strike outsiders as very blunt when it involves, for example, initiating a one-on-one meeting with someone one does not know and who is not expecting the meeting request.

Only one Microsoft informant reported making hardcopies of her calendar and bringing them to a meeting. When people are away from their desks (e.g., in a meeting) and need to schedule something, common phrases are "Sched plus me" or "Plus me." Someone later finds an available time and sends out SCHEDULE+ mail.

A conference room has a calendar that is viewed and scheduled like any other. The conference room appears in the To field of the meeting invitation message. If meeting location information is omitted from the Subject line (which is read by SCHEDULE+ when the request is loaded into a person's calendar), a recipient can examine the To field to see which conference room has been "invited to" or booked for the meeting.

At Sun, the most commonly used privacy setting is also the application default, but in this case the default, world browse, allows others to see the descriptions of appointments as well as their times. Descriptions of private appointments, such as interviews and medical appointments, must therefore be "blacked out" individually. Employees use CALENDAR MANAGER to check weekly calendars to determine availability for formal meetings and for informal drop-ins. Seeing the content of a meeting can be useful: It can indicate whether a person is in their office or where they are and when they might be back, how interruptable they might be, how "reschedulable" a meeting might be in case of a conflict, and so forth. Browsing has other uses: Managers report locating subordinates and vice versa. "Browsing VP (Vice President)'s calendars is a lot more interesting," said one first-level manager, although perhaps not all VPs set their calendars to world browse.

To initiate a group meeting, Sun interviewees generally send regular email listing a possible time. Many are either unaware of the various ways a meeting template or drag and drop icon can be generated, or they find them inconvenient. But some of them take the trouble to type information in meeting template format so recipients can drop it into their calendars for automatic loading. People are more likely at Sun than at Microsoft to bypass the system to set up a one-on-one meeting, although they generally enter such appointments in their calendars after scheduling them.

Our Sun informants rarely print out calendars either (although we have evidence that managers and executives are more likely to do so). If a need to arrange a meeting arises, someone typically takes responsibility for setting it up, often in response to a request to "Browse my calendar" or "Browse me." Most groups use a separate application to schedule conference rooms, but special equipment in high demand is given a calendar for people to "browse" to find available time. 


\section{Factors Contributing to Successful Adoption}

Many factors can contribute to a pattern of application use in a particular setting, not all of which may be clear to participants and thus not recovered from interviews. However, our interviews addressing calendar use in 15 groups in two organizations yielded evidence of conditions and patterns different from those prevailing ten years ago in similar environments. These differences, which plausibly contribute to the improved reception that meeting scheduling now enjoys, include:

- the infrastructure, including network, software, support, and behavior

- expanded application functionality

- the versatility and ease of access provided by graphical interfaces

The adoption of communication technologies occurs over time. Often a critical mass of use must be reached before a technology provides a net benefit (Markus, 1987). Virtually all group members must adopt some technologies before they are useful. Group calendaring has been described as one such application by researchers (Ehrlich, 1987b) and by some of our informants. How does one obtain universal compliance? The claim that management must mandate use has been made for groupware, but as noted in the introduction, groupware such as meeting scheduling may not be considered important enough for management to pressure people to do the substantial work of keeping their calendars on-line. Another approach found in the literature of success cases is that of the fervent product evangelist who persuades all group members to try the software, thus obtaining the critical mass that brings real benefits to the group.

Did we find management mandates or evangelist networks in our 16 groups? We found a few cases in which group leaders strongly encouraged group use, and we interviewed one self-described evangelist. But these did not explain the widespread use of meeting scheduling. Instead, we found widespread reports of peer pressure. Two notable aspects of this pressure were:

- aspects of the product interfaces that facilitate the delivery of peer pressure

- adoption was described several times as following a bottom-up pattern from developers to managers and administrative assistants ("admins"). Pressure on recalcitrant users could eventually come from every direction-managers, admins and peers - and adoption became nearly universal.

We now consider in turn each of the five bulleted items above.

\section{Infrastructure}

Both sites have organization-wide platforms supporting the calendar and scheduler. All employees with PCs (Microsoft) or workstations (Sun) were networked.

Ten years earlier, this was not true in large high-tech companies. Part of a company might rely on a mainframe, elsewhere minicomputers - the platform for groupware of that period-were in use, and PCs made inroads but were difficult to network. Email could not span these environments, much less meeting scheduling software. Members of a team might share a platform, but many meetings (other 
than regularly scheduled group meetings) included people from other groups; therefore scheduling software could not routinely replace traditional scheduling methods. Our informant who did not use the calendar was outside development, in corporate marketing; many of her meetings involved people external to the company whom the software could not reach. Ten years earlier, most developers were in comparable positions with respect to other groups within the company.

Both sites also provide strong technical support in installing and maintaining the software. This arose in several interviews. Group calendaring over a large clientserver network is technically challenging. The failure of two releases of CALENDAR MANAGER was attributed by one informant to reliability problems, and occasional losses persist:

About 2 years ago <sigh/laugh>, I lost all my data. And they had no idea what happened, it just was gone. So I mean all my personal data was gone too...I felt so stupid that I had to go back and call all these people. I lost all my birthday data, ... my work data ... I was lost, because I rely on my calendar to tell me where I am supposed to be and what I am supposed to be doing. I really was relying on it. When I lost the data, I had to go around and try to find it, or I'd get calls "where are you?" "well I am right here, where am I supposed to be?" So, you know it was an embarrassing time.

Clearly not much of this will be tolerated in a product.

Finally, although difficult to measure, behavior has changed. People are much more comfortable with technology and are heavier users of it now than was true ten years ago, even in engineering environments. Almost everyone in these settings can be counted on to read email regularly, one element of what Markus (1987) calls "message discipline," an important factor in promoting use. The casual use of phrases such as "Sched plus me" and "Browse me" is further evidence of structuration or an evolution of the culture around the technology. A "behavioral infrastructure" was also in place.

Functionality

Another change in ten years is product maturation. SCHEDULE+ requires about $2 \mathrm{M}$ RAM on each client and 5M disk space between client and server; CALENDAR MANAGER requires about 2-4M RAM and 1M or more disk space. Unlike earlier products and research systems, these applications were strongly integrated with desktop environments, email and corporate on-line rolodexes. The tight coupling with email was singled out as a factor in early adoption.

Conference room availability was sometimes described as the most critical aspect of scheduling a meeting, so the ability to schedule rooms or equipment through the system is another example of a feature that promotes use.

Both systems provide a broad range of privacy options. Informant attitudes toward privacy differed, so this flexibility could be important.

Seemingly minor features were often praised: the diverse mechanisms for reminding oneself of an upcoming event or a new release that increased the ways to 
define recurring meetings, such as 'Mondays, Wednesdays and Fridays at 9.' ('Feature creep' may afflict calendars but does not yet seem a problem.)

One admin had used CALENDAR MANAGER for six years to keep calendars for managers, but only started keeping her own three years earlier:

[I started using it for myself] when I realized all the things it could do ... I don't remember it being that sophisticated before ... I mean it is an incredible thing, I mean now you can show multiple calendars on top of each other. So it's really just gotten so good, it's become a valuable scheduling tool ... I would say that with all of the increased capabilities of CALENDAR MANAGER that a lot more people have started to use it and realized that it's a very helpful thing.

Versatility and Ease of Access

In discretionary use situations, ease and enjoyment are powerful motivators. (If the MACINTOSH did not prove this, the success of interfaces such as EUDORA and MOSAIC have.) With the applications studied, many calendar and scheduling features are easily accessed and many tasks can be carried out multiple ways, with one or another way more natural depending on the context. With the earlier generation of schedulers, obtaining information was too time-consuming to promote some of the casual uses of calendar information that $\square$ we observed. Even when unrelated to meeting scheduling, these promote the prerequisite on-line calendar use; they contribute to "calendar discipline," the behavioral infrastructure.

We found evidence that for applications that are only indirectly tied to people's principal work missions, interface transparency and efficiency are particularly important. Where a feature is even slightly obstructed, it can go unused. For example, CALENDAR MANAGER users greatly appreciate receiving invitations in the form of a "meeting template" which can be dropped into their calendar. Yet many message senders do not use either of the ways to drop a meeting template into their invitation, each of which involves an extra step or two: Instead they take more time to type an invitation character by character to conform to the message template.

One developer pithily summarized his attitude: "walk arounds are work arounds." If he felt blocked by the application, rather than looking for a way to work around the problem, he would arrange meetings "off-line," on foot.

An interface change that was widely praised at Microsoft was allowing users to enter employee's actual names, in contrast to their email aliases. At Sun, employees' calendars had to be identified by name and host machine. The integrated on-line rolodex renders this only a minor inconvenience; nevertheless, this was a common source of complaints, suggesting that small interface details are salient to users who may not be strongly committed to an application.

Universal Adoption and Peer Pressure

"The only way that makes it useful is that everyone is using it." - Product marketing representative 
Use was almost universal in the groups sampled, with some people unaware of anyone who did not use it. A Sun developer remarked "More people are running this stuff now, absolutely. It's probably almost $100 \%$ at this point." An admin estimated "Ninety-eight percent. I think that it probably went from like 50\% when I [first] really started using it." Another developer said:

The numbers have gone up dramatically. I think now ... everybody uses it, anybody who has a workstation uses it. It's hard to find someone who doesn't use it ... It'd probably be the janitor or someone like that because they just don't have access to the equipment. Everybody ... even non-technical people use it.

How did use become so widespread? When asked directly, some reported peer pressure to keep calendars on-line, some admitted exerting such pressure, others said they did not notice pressure, but might contradict themselves in subtle or not so subtle ways. "One person in this group refuses to use it and it drives people nuts," said a Microsoft designer. A Microsoft middle manager described a higher-level manager: "She doesn't use SCHEDULE+. That's a source of frustration." An admin at Sun reported telling the only non-user he knows: "Everyone on the team is available at this date and time, except I don't know whether you are even in your office." A Sun human factors engineer who said "I don't think that there's any pressure to use it" also said:

Where I find that things become annoying is that a lot of people will call for meetings without using the calendar appointment embedded in their message ... then I've got to go into the CALENDAR MANAGER and schedule an appointment and it seems like a waste of time. And that happens all the time.

(He noticed and pointed out the contradiction himself.) A manager echoed this:

I find it a real pain in the neck [when someone doesn't include the appointment icon]. It amazes me ... that people send out appointment notices that don't include them-irritating. They're lazy. I know they use CALENDAR MANAGER so I mean ... they haven't figured out how to do it, or it's just not important to them to think of that.

In environments where "sched plus me" or "browse me" are common phrases and future meeting dates are not set on the spot, non-users will quickly feel left out. In fact, they miss out. "Individuals were embarrassed about not having shown up to meetings. There was peer pressure to start." (Microsoft.) "Once people start sending you appointments then that behooves you to look at your calendar some and also suggests, 'well gee I've got appointments that other people are giving me, so maybe I should do the same." (Sun.)

Other forms of inducement include sending out lists of holidays or birthdays formatted to be dropped into calendars (which is a recent practice at Sun).

The application design can itself contribute to social pressure. Recognizable message templates remind non-users of a benefit they may be missing. Integration with email enables the technology to deliver peer pressure. A project manager at Microsoft reported "Some people don't look at my SCHEDULE+ [calendar] to see if I'm available. I will press decline, and say please see my SCHEDULE+." The recipient is left with little option other than to use the software. 


\section{The Adoption Trajectory}

No single adoption pattern fit every group. A few reported pressure from managers, and at Microsoft group by group adoption occurred. Several developers and managers reported that developers-individual contributors - were the first adopters, exerting pressure on peers and then on managers and admins, with pressure continuing up the managerial ladder. Once admins adopt meeting scheduling they can exert pressure in all directions. (It may be a significant factor that admins appear to wield more influence than secretaries did ten years ago.)

People expressed a variety perceptions about adoption. A Microsoft program manager said: "I resisted for six months. When I had people under me, I needed it. Now I can't live without it." A Sun corporate marketing representative, who initially used CALENDAR MANAGER but has since stopped, reported:

[In] Sun culture, engineers [say] 'You are not on CALENDAR MANAGER?' They're just shocked that you don't use your own technology ... I don't think marketing management uses [CALENDAR MANAGER].

A Microsoft trainer reported that "the first groups to get up to speed were the admins." Similarly, a Sun product marketing representative said:

I think that most people keep a CALENDAR MANAGER because of the browsing capabilities.... When we are trying to set up appointments, admins ... rely on being able to look at other peoples' calendars to see when they are available.

A Sun developer described a bottom-up adoption pattern:

Over time, they (managers) started to use it, they started seeing the benefit of using it ... but initially I can't say there were a lot of people using it. Initially ... it was mostly developers, then you start getting a scattering of managers, I bet you now we could go and browse Scott McNeely's (Sun CEO) calendar if we wanted to, so now all the way to the top ... now everybody.

However, a Sun technical writer reported a different experience: "pressure was from management, not coworkers. The attitude is "Get into the 90s.",

\section{Conclusion}

In these large organizations, groupware can succeed without mandated use, helped by the congenial functionality and interface features associated with discretionary use individual productivity tools. The features, some of which provide individual benefits (e.g., meeting reminders), may attract a critical mass of users, after which technology-abetted social pressure by peers and others extends use.

We do not say groupware use always follows this pattern; we found examples of mandated use, albeit from low management levels. Mandated use can clearly lead to or facilitate adoption. In addition, in contrast to these large established organizations, we have seen some new, small enterprises that organize themselves around groupware technologies, a de facto mandate. More study of the successful adoption of a variety of applications in diverse settings is needed to assess the significance and representativeness of these and other factors. 


\section{Acknowledgments}

We are grateful to interview participants at both sites; to Ellen Isaacs for her enthusiasm and intellectual and operational support for the project; to Rick Levenson, Mark Simpson, Susan Denning, and the Microsoft Usability Group for their support; and to Susan Ehrlich Rudman, Steven E. Poltrock, and Lynne Markus for comments and help in identifying details of calendar use in different places and times. This material is based in part upon work supported under a National Science Foundation Graduate Research Fellowship awarded to the second author.

\section{References}

Beard, D., Palaniappan, M., Humm, A., Banks, D. and Nair, A. (1990): “A visual calendar for scheduling group meetings.” Proc. CSCW'90, 279-290. New York: ACM.

Butterfield, J., Rathnam, S. and Whinston, A. B. (1993): "Groupware perceptions and reality: An e-mail survey." Proc. 26th Annual HICSS, 208-217.

Ehrlich, S. E. (1987a): "Social and psychological factors influencing the design of office communication systems. " Proc. CHI+GI' 87 , 323-329. New York: ACM.

Ehrlich, S. E. (1987b). "Strategies for encouraging successful adoption of office communication systems." ACM Transactions on Office Information Systems, 5, 340-357.

Grudin, J. (1988): "Why CSCW applications fail: Problems in the design and evaluation of organizational interfaces." Proc. CSCW'88, 85-93. New York: ACM. Extended version in Office: Technology and People, 4, 3, 245-264.

Grudin, J. (1994): "Groupware and social dynamics: Eight challenges for developers." Communications of the ACM, 37, 1, 92-105.

Grudin, J. and Poltrock, S. E. (1990): "Computer Supported Cooperative Work and Groupware." CHI'90 Tutorial Notes. NY: ACM.

Markus, M. L. (1987): “Toward a 'Critical Mass' Theory of Interactive Media.” Communication Research, 14,5, 491-511.

Markus, M. L. (1995). "Disimpacting use: How use of information technology creates and sustains organizational transformation." Working paper, February 1995.

Markus, M. L. and Connolly, T. (1990): "Why CSCW applications fail: Problems in the adoption of interdependent work tools." Proc. CSCW'90, 371-380. New York: ACM.

Orlikowski, W. (1992): "Learning from Notes: Organizational issues in groupware implementation. Proc. CSCW'92, 362-369. NY: ACM.

Woitass, M. (1990): "Coordination of intelligent office agents - applied to meeting scheduling." In S. Gibbs and A. A. Verrijn-Stuart (eds.), Multi-User Interfaces and Applications, pp. 371387. Amsterdam: North Holland. 